\title{
Detection and simultaneous confirmation of Methicillin-Resistant Staphylococcus aureus by an automated Real-Time PCR method with strain differentiation by analysis of DNA melting curve characteristics
}

\author{
Aus Molan \\ Department of Research and in-vitro Diagnostics/Abacus ALS/New Zealand
}

\begin{abstract}
Methicillin-resistant Staphylococcus aureus (MRSA) continues to be a burden to the global healthcare system. Locally, the prevalence of MRSA in New Zealand has increased significantly over the last 10 years underscoring the urgency to control the colonisation and spread of MRSA. Following on from a previous multi-centre study looking at MRSA colonisation in the nares and throats of a New Zealand population using a PCR method, this study aims to determine the usefulness of DNA melting curve analysis in the differentiation of MRSA strains. Specifically investigated is a possible relationship between the isolate DNA melting temperature $\left(T_{M}\right)$ and MRSA strain. Bacterial typing can be used to confirm or refute the relatedness of isolates and to plan MRSA control programmes and while the determination of MRSA strains is currently done using staphylococcal protein A gene typing based upon repeat pattern analysis and pulse-field gel electrophoresis, these processes are often time consuming, require specialist skills, and are performed at reference laboratories. In this study, 10 MRSA isolates where detected from a total of 270 volunteers using the Roche LightCycler MRSA Advanced Test. It was found that the WSPP was the most frequently isolated strain. In addition, by using the DNA melting temperatures, an apparent link was found: the WSPP strain had the highest $T_{M}$, followed by the AK3 strain, and lastly, the WK/AK1 strain had the lowest $T_{M}$. Moreover, no variation in $T_{M}$ was observed between different volunteer isolates of the same strain type (WSPP, AK3, and WK/AKI displayed consistent $T_{M}$ values of $59.6^{\circ} \mathrm{C}$, $58.1^{\circ} \mathrm{C}$, and $57.7^{\circ} \mathrm{C}$, respectively). The significance of swiftly determining the MRSA strain is somewhat understated. From an infection control/epidemiology perspective, it provides information on origin and mutation shift of the pathogen, helping in the control of MRSA epidemics, thus contributing to our knowledge of strain dynamics. More importantly perhaps, it provides information on determining the course of treatment for the patient as strains differ in their antimicrobial susceptibility patterns. While it is too early to draw conclusions from this data, it certainly warrants further investigation.
\end{abstract}

Keywords: Methicillin-Resistant Staphylococcus aureus, MRSA, Real-Time Polymerase Chain Reaction, PCR, nucleic acid thermodynamics, DNA melting temperature, infection control.

\section{INTRODUCTION}

Staphylococcus aureus is an opportunistic pathogen carried as a commensal organism on the skin and in nares of approximately $30 \%$ of the normal population [1]. S. aureus is responsible for a broad range of clinical infections, most notable of which are cases of bacteraemia and endocarditis [2]. Methicillin-resistant $S$. aureus (MRSA) was first described in 1961, with the first hospital outbreak reported in 1963 [3]. Healthcare associated (HA) infections caused by MRSA have become an important issue for healthcare facilities worldwide due to high rates of infection, mortality, and high costs of treatment [4]. With $20 \%$ of invasive MRSA infections resulting in death, the failure to decrease HA-MRSA poses a serious threat to patients' health [5]. Cases of community-associated MRSA (CA-MRSA) infections were first reported in the late 1980s and early 1990s [6]. More importantly, CA-MRSA has spread in the past few years [7], feeding the pipeline of infection in hospitals [5], and underscoring the need for comprehensive infection control programs. During 2005, it was estimated that invasive MRSA infections in the United States resulted in almost 19,000 deaths, a figure higher than that for HIV/AIDS [5].

Based on data gathered by the Institute of Environmental Science and Research Limited (ESR, Porirua, New Zealand), the prevalence of MRSA among the population in New Zealand has approximately doubled over the last 10 years (2003-2012), with the largest single-year increase (37\%) occurring between 2010 and 2011 [8]. The strains of MRSA associated with CA infections frequently belong to lineages separate from MRSA associated with HA infections [9]. However, this distinction is blurring with some community-associated MRSA (CA-MRSA) strains now also causing healthcare-associated infections. According to Heffernan and Bakker [8], the six strains collectively responsible for $90.2 \%$ of MRSA isolation in New Zealand in 2012 (AK3 MRSA, WSPP (Western Samoan Phage Pattern) MRSA, WR/AK1 MRSA, EMRSA-15, Queensland clone MRSA and USA300 MRSA) are usually considered CA-MRSA. The EMRSA-15 strain was the only HA- 
MRSA strain and accounted for just $8.7 \%$ of MRSA isolated [8]. The current predominance of CA-MRSA strains indicates that MRSA is more commonly transmitted and acquired in the community in New Zealand than the in healthcare facilities. This highlights the importance of strain dynamics and the need to type MRSA strains to track spread and infection within both the community and healthcare settings.

Effective control measures for reducing the hospital and community reservoirs of MRSA depend primarily on knowing the risk factors of the exposed population for MRSA colonisation [5, 10]. The acquisition of MRSA has many risk factors associated included prolonged hospitalisation, preceding antimicrobial therapy, use of invasive procedures, treatment in an intensive care unit, surgical procedures and close proximity to a patient colonised or infected with MRSA [4, 6, 10]. Furthermore, heavy dependency, i.e., patients requiring considerable nursing and other support is another risk factor that should be considered [10].

Control and prevention of MRSA infection or colonisation may include analysis of isolates by discriminatory bacterial DNA typing. Bacterial strain typing distinguishes epidemiologically related or clonal isolates from unrelated isolates. Typing plays an important role in understanding the epidemiology of MRSA and evaluating the effectiveness of infection control and antimicrobial prescribing measures [11]. Several investigators have demonstrated the superiority of macrorestriction analysis by pulsed-field gel electrophoresis (PFGE) for strain characterisation, when compared with other techniques [10-12]. This method provides highly reproducible restriction profiles that typically show distinct, well-resolved fragments representing the entire bacterial chromosome and is considered to be the method of choice for DNA fingerprinting of MRSA and other bacterial pathogens $[11,12]$.

Laboratory-based screening for MRSA colonisation in patients and healthcare workers remains a cornerstone of infection control measures to limit the spread of MRSA [13]. In 2008, Roche Molecular Systems (Switzerland) introduced the LightCycler (LC) MRSA Advanced Test, an in vitro diagnostic PCR method for the rapid detection of MRSA colonisation to aid in the prevention and management of MRSA infections in healthcare settings. The test targets the integration site of the SCCmec cassette into the $S$. aureus chromosome with melting point analysis of the PCR product $[14,15]$. The assay relies on three major processes: preparation of the specimen by mechanical lysis of the bacterial cell wall; detection of PCR amplified target DNA by specific hybridisation probes; and melting peak analysis and automated result generation. This PCR assay is performed on the LightCycler 2.0 (LC, Roche, Applied Science) instrument, an extremely fast thermocycler with on-line fluorescence detection. The processed samples and the amplification mixture containing hot-start Taq polymerase are placed in LC Capillaries in which PCR amplification occurs. Each LC Test reaction contains an internal control, which is designed to control for specimen inhibition, and to monitor reagent integrity. The AmpErase (uracil-N-glycosylase) enzyme included in the LC Test recognises and catalyses the destruction of DNA strands containing deoxyuridine but not DNA containing deoxythymidine. Since amplicons produced with the LC Test contain deoxyuridine, potential amplicon contaminants are eliminated during a prolonged heating step performed prior to the start of PCR amplification. A target sequence in a plasmid is simultaneously amplified in the positive control. The positive control is intended to monitor reagent failure and is included into each run. Each run also includes a negative control used to detect reagent or environmental contamination by MRSA DNA. MSRA and internal control amplicons are detected by fluorescence using a specific pair of hybridisation probes. The probes attach to a specific internal sequence in the amplified fragment and are positioned in close proximity to one another and upon excitation, a fluorescence signal of a specific wavelength is emitted from these bound probes using a process called Fluorescence Resonance Energy Transfer (FRET). The emitted light is measured by the LightCycler 2.0 Instrument. Two different detection channels detect, in parallel, the MRSA and the internal control specific amplicons thus making differentiation possible. After completion of the real-time PCR process, the LightCycler 2.0 Instrument automatically performs a melting peak analysis. The single stranded DNA amplicons with bound hybridisation probes are subjected to increasing temperatures. When the PCR products reach a specific temperature, one of the two bound hybridisation probes melts off, resulting in a loss of florescence signal. This decrease in the fluorescence signal is temperature specific and results in melting peaks that are used to identify and differentiate MRSA and internal control specific amplicons. Real-time PCR amplification of MRSA DNA is monitored via LC Software version 4.1. After completion of the run, identification of the melting peaks is performed using $\mathrm{T}_{\mathrm{M}}$ bars in the LC software.

The aim of this study is to determine if particular MRSA strains occupy certain distinguishable DNA melting point curves thereby establishing a link between the DNA melting temperature and MRSA strain. This is carried out by comparing the melting point peaks produced after PCR analysis of each sample with the staphylococcal protein A ( $s p a)$ gene typing (usually assigned to a particular strain type). The PCR method used is the Roche LC MRSA Advanced Test while the spa gene typing was determined using a method adapted from that described by Strommenger et al. [16]. 


\section{II.1 Study design}

\section{MATERIALS AND METHODS}

In a previous study conducted by Molan et al. [17], 810 specimens were obtained from 270 eligible volunteers between June and December 2011. Subject samples were collected at nine sites across New Zealand. Specimens were tested at one site in Palmerston North, New Zealand. The volunteers consisted of the following: patients from hospital non-intensive units; medical laboratory staff; and hospital medical staff including nurses, physicians, infection control and administration staff. All volunteers were $\geq 16$ years of age and provided written informed consent to participate in the study. The isolation, confirmation, and antimicrobial susceptibility testing of the MRSA isolates used in the current study have been previously described [17].

In this study, the MRSA positive samples isolated from the above mentioned study underwent further investigation using the Roche LC system with DNA melting temperature analysis. The Roche LightCycler MRSA Advanced Test was performed according to the manufacturer's instructions (Roche Diagnostics, Switzerland). This assay targets the integration site of the SCCmec cassette into the $S$. aureus chromosome. Essentially, the LC PCR assay is performed on the LightCycler 2.0 instrument and has been validated with three transport media (Liquid Stuart, and Amies gel with or without charcoal). Detection of MRSA with the LightCycler MRSA Advanced Test relies on 3 major processes: sample preparation by mechanical lysis of the bacterial cell walls; PCR amplification of the target DNA and detection by hybridisation probes; and, result generation of the PCR assay after melting peak analysis. Electronic timers were used to monitor the start and finish times for sample preparation, working master-mix preparation, and the PCR preparation for the LC PCR method.

\section{II.2 Sample preparation}

Each run consisted of 32 samples run in parallel (30 volunteer samples plus positive and negative controls). Swab heads were cut into lysis tubes and subjected to heating $\left(95^{\circ} \mathrm{C}\right.$ for two minutes in a dry blcok) to inactivate bacteria. The lysis tubes were then transferred to the MagNa Lyser instrument (Roche Applied Science, Switzerland) for 70 seconds at a speed of 5,000 rpm where mechanical lysis of the bacterial cell wall takes place, resulting in a crude lysate preparation. After a rapid centrifugation step at room temperature $(500 \mathrm{~g}$ for one minute) to spin down the swab fibres and glass beads, the processed specimens were transferred to a $20 \mu \mathrm{L}$ capillary containing the reaction and MRSA detection mix. The capillaries were then placed in the LightCycler 2.0 Instrument for PCR analysis.

The Working Master Mixture was prepared by adding 350 $\mu \mathrm{L}$ of the MRSA Reaction Mixture to $175 \mu \mathrm{L}$ of the MRSA Detection Mixture giving a total volume of $525 \mu \mathrm{L}$. The master mix was transferred in $15 \mu \mathrm{L}$ aliquots to pre-positioned LC capillaries on the LC Carousel. Subsequently, $5 \mu \mathrm{L}$ of clear sample supernatant, positive control, and negative control, was added to the capillaries.

\section{II.3 Reagents}

The Lysis Reagent contained tris buffer, EDTA, <15\% silica beads, and $0.09 \%$ sodium azide. The MRSA Reaction Mixture contained tris buffer; $<0.1 \%$ magnesium chloride; potassium chloride; $<0.001 \%$ dATP, dCTP, dGTP, dUTP; $0.19 \%$ octyglucoside; $0.03 \%$ mammalian BSA; <0.1\% FastStart Taq DNA polymerase (microbial); $<0.01 \%$ amperase (uracil-N-glycosylse) enzyme (microbial); and $0.09 \%$ sodium azide. The MRSA Detection Mixture contained 0.05\% Brij@ 35 solution, tris buffer, <0.01\% EDTA, < $0.01 \%$ MRSA primers, < $0.01 \%$ fluorescent-labeled oligonucleotide probes, specific for MRSA and the MRSA internal control, $0.09 \%$ sodium azide, $0.001 \%$ poly rA RNA (synthetic), $<0.001 \%$ non-infectious plasmid DNA (microbial), containing MRSA primer binding sequences and a unique probe binding sequence. The MRSA positive control contained tris buffer, $<0.01 \%$ EDTA, $0.002 \%$ poly rA RNA (synthetic), $0.09 \%$ sodium azide, $<0.001 \%$ non-infectious plasmid DNA (microbial) containing MRSA sequences. For the negative control, a sterile swab head was used and processed in the same routine as a volunteer swabs.

\section{II.4 Amplification}

This assay uses real-time PCR amplification of MRSA DNA, and fluorogenic target-specific hybridization probes for detection of amplified DNA. Each LC PCR test reaction mixture contained an internal control to detect specimen inhibition and to monitor reagent integrity. The internal control was a plasmid amplified with primers used for amplifying the MRSA target. After the sample preparation protocol outlined above, the capillaries were sealed and the carousel centrifuged in the LC Carousel Centrifuge before being transferred to the LC instrument. The cycling protocol (Fig. 1) consisted of one 10 minute cycle at $95^{\circ} \mathrm{C}$ followed by 45 cycles consisting of denaturation for 10 seconds at $95^{\circ} \mathrm{C}$, annealing for 10 seconds at $55^{\circ} \mathrm{C}$, and elongation for 15 seconds at $72^{\circ} \mathrm{C}$. The temperature ramp rate was set at $20^{\circ} \mathrm{C}$ per second. After the final cycle, the capillaries were cooled for $2 \mathrm{~min}$ at $40^{\circ} \mathrm{C}$. 


\subsection{Automated result generation}

The fluorescence of each capillary is measured at $530 \mathrm{~nm}, 550 \mathrm{~nm}, 610 \mathrm{~nm}, 640 \mathrm{~nm}, 670 \mathrm{~nm}$, and $705 \mathrm{~nm}$. A second aspheric lens transmits the signal of each detection channel to a photohybrid for final evaluation before being the signal is sent to the LC instrument via a serial interface. The Real-time PCR amplification of MRSA DNA was monitored via LightCycler Software version 4.1. After a visual identification of the melting peaks was performed using the $T_{M}$ bars in the LightCycler software, test results were transferred to a dedicated interpretation tool (Micro Analysis Software) and a report of the results was generated.

\section{II.5 Staphylococcal protein A (spa) typing}

MRSA isolates were sent to the Institute of Environmental Science and Research, Porirua, New Zealand (ESR) for staphylococcal protein A gene (spa) typing. ESR sequence the spa gene using a method adapted from that described by Strommenger et al. [16]. spa sequences were analysed using Ridom StaphType software version 2.0.3 (Ridom GmbH, Würzburg, Germany). In this system, each repeat sequence is assigned a unique identifier and the combination and order of repeats corresponds to a unique spa type. The clonal relatedness of different spa types can be identified and clustered using the BURP (based on repeat pattern) algorithm.

\section{RESULTS}

Figure 2 displays the fluorescence versus cycle number curves for the runs containing the 10 MRSA positive clinical samples. Also displayed are the melting point peaks for these samples. While there is some variation in the melting temperature curve points of the isolates, all the WSPP strains isolated demonstrated the highest $\mathrm{T}_{\mathrm{M}},\left(59.6^{\circ} \mathrm{C}\right)$, followed by the AK3 strains $\left(58.1^{\circ} \mathrm{C}\right)$, and lastly, the WK/AK1 strains had the lowest $\mathrm{T}_{\mathrm{M}}$ $\left(57.7^{\circ} \mathrm{C}\right)$. The melting temperature peak values for all samples including the positive and negative controls were within the specified acceptable melting temperature range stipulated for the LC MRSA method $\left(57-62^{\circ} \mathrm{C}\right)$.

Table 1 summarises the antimicrobial resistance, melting temperatures $\left(T_{M}\right)$, and strain variation between the MRSA isolates. The WSPP strain was the most frequently isolated strain, followed equally by the AK3 and WK/AK1 strains. Three samples contained the t5677 spa type which is not related to any identifiable strain type. MRSA isolates were resistant to: penicillin (100\%), erythromycin (10\%), clindamycin (10\%), ciprofloxacin $(0 \%)$, mupirocin $(20 \%)$, rifampicin $(0 \%)$, gentamicin $(0 \%)$, tetracycline $(0 \%)$, fusidic acid $(20 \%)$, and co-trimoxazole $(0 \%)$.

The technical hands on time as well as the mean time to result for the Roche LightCycler MRSA advanced test are presented in Table 2. The labour involved was documented for 30 specimens and then averaged for a per sample value. Calculations included: lysate preparation; working master mix preparation; and specimen and control PCR preparations. Regardless of the result, the average personnel hands-on-time was 1.65 min per sample for the LC PCR assay.

\section{DISCUSSION}

In the present study, the WSPP strain was the most frequently isolated strain, followed equally by the AK3 and WK/AK1 strains. While isolated in low numbers, the results are consistent with a previous report [18]. However, it seems that the dominance of the WSPP clone in New Zealand is diminishing. Reports have documented that the prevalence of AK3 MRSA has increased each year since 2005, and from 2010 to2012 this strain accounted for the highest proportion (29.0\% and $47.2 \%$, respectively) of MRSA isolations [8, 19]. All the MRSA strains isolated in this study had type IV SCCmec elements. In New Zealand, it is now apparent that type IV SCCmec strains dominate MRSA isolations, and that this has been the case since 1995 [18]. Since then, these strains have comprised on average almost three-quarters of all isolates per year, with a mean of $74 \%$ [18, 19].

Although WSPP, AK3, and WR/AK1 MRSA strains are considered community-associated, in this study they were all isolated from laboratory health workers and a hospital patient. Similar findings have been reported by Smith and Cook [18]. Volunteers where not categorised according to where they acquired MRSA therefore some participants who may have acquired MRSA in the community were categorised as hospital patients or staff. This is supported by the fact that the strains isolated in this study were not multi-resistant, a feature typical of community-associated MRSA [8, 19]. Additionally, as MRSA strains become progressively common in a variety of settings, the use of restrictive terms like HA-MRSA and CA-MRSA will become less meaningful as spread between all populations occur. The potential to recover an increasing array of SCCmec types, especially from community-occurring MRSA strains, clearly exists [20].

While there is some variation in the melting temperature curve points of the isolates, a link between the MRSA strain and the melting temperature is apparent: the WSPP strain had the highest $\mathrm{T}_{\mathrm{M}}$, followed by the AK3 strain, and lastly, the WK/AK1 strain has the lowest $T_{M}$. Moreover, no variation was observed in the $T_{M}$ values between different volunteer isolates of the same strain- that is to say, the $\mathrm{T}_{\mathrm{M}}$ values for the all the isolated strains (WSPP, AK3, and WK/AK1) were consistent at $59.6^{\circ} \mathrm{C}, 58.1^{\circ} \mathrm{C}$, and $57.7^{\circ} \mathrm{C}$, respectively. This suggests 
that the melting temperature generated by the LC method may indicate the strain of MRSA. Being able to rapidly generate such knowledge will aid in our understanding of the epidemiology of MRSA and will also serve a purpose for evaluating the effectiveness of infection control and antimicrobial prescribing measures [11]. While antimicrobial susceptibility testing is routinely performed by clinical microbiology laboratories on most bacterial isolates to aid in the identification of new or unusual patterns of antibiotic resistance, the results can only raise the suspicion of an outbreak or the presence of a new strain. This is due to the fact that antibiotic susceptibility testing has relatively limited use in epidemiological studies because of phenotypic variation as observed in this study, and because antibiotic resistance is affected by selective pressure in hospitals [10]. Therefore strain typing provides a more robust epidemiological tool for MRSA infection in the population. Certainly, using melting curve analysis, Chan et al. [21] were able to report on a probe-target mismatch as demonstrated in one of their evaluations of this LC assay. They reported a shifted melting peak phenomenon which was also present in Hong Kong, where a similar proportion of MRSA also have a probe target mismatch. In Hong Kong, the mismatch was due to a single nucleotide polymorphism in ST45-MRSA-IV, with the uncommon finding of a porcine strain ST398 $\left(\mathrm{T}_{\mathrm{M}}>60^{\circ} \mathrm{C}\right)$ and a ST672-MRSA-IV [21].

From this study, it was calculated that the average personnel hands-on time per sample for the PCR method was 1.65 minutes. Similar findings have been published by Peterson et al. [14]. While average handsone time figures as low as 1.2 minutes per sample have been previously reported for a chromogenic culture method [17], the significant difference between culture and PCR is the time to result for each method. By using the Roche LC PCR assay, laboratories have the ability to obtain results within two to three hours. Realistically, in a clinical setting, where batch processing of samples for PCR assays is performed once a day, the turnaround time for the PCR method may equate to 10-15 hours, a figure previously reported [22]. Even if this was the case, the culture method is still much slower. According to Valle et al. [23], reliable and rapid detection of MRSA-colonised patients is essential for the successful prevention and control of MRSA outbreaks in hospital, and hence for overall patient care and hospital infection control. The Roche LightCycler MRSA assay's speed (2 hours versus an average reported figure of 24-48 hours for the culture method) gives it a critical advantage over culture in this respect. Previous studies have documented the importance of timely identification of MRSA colonisation [15, 23, 24]. This allows the isolation and treatment of MRSA affected patients and hence is critical for the prevention of MRSA outbreaks. A study by Cunningham et al, [25] demonstrated a reduction in the transmission of MRSA incidence from 13.9/1,000 patient days (under culture method) to 4.9/1,000 patient days under PCR screening. Additionally, the PCR method used in this study required only four hours of staff training with minimal expertise required to perform the assay

An obvious limitation of this study is the low number of positive MRSA samples collected from volunteers. In the future, a larger sample size would need to be accumulated and analysed to produce more statistically significant sets of data.

\section{CONCLUSIONS}

From this study, it can be concluded that the Roche LightCycler MRSA Advanced Test is another accurate and rapid method in the detection of MRSA colonisation. The test also has the added ability to analyse DNA melting curve characterises. This feature has been shown to have potential in presumptively identifying the MRSA strain with particular strains occupying certain temperature ranges. This is important to our understanding the epidemiology of MRSA and evaluating the effectiveness of infection control and antimicrobial prescribing measures. While more research is needed in this field, DNA melting curve analyses may prove especially valuable when a clear relationship is established between strain variation and nucleic acid thermodynamics of MRSA. 
VI. FIGURES AND TABLES

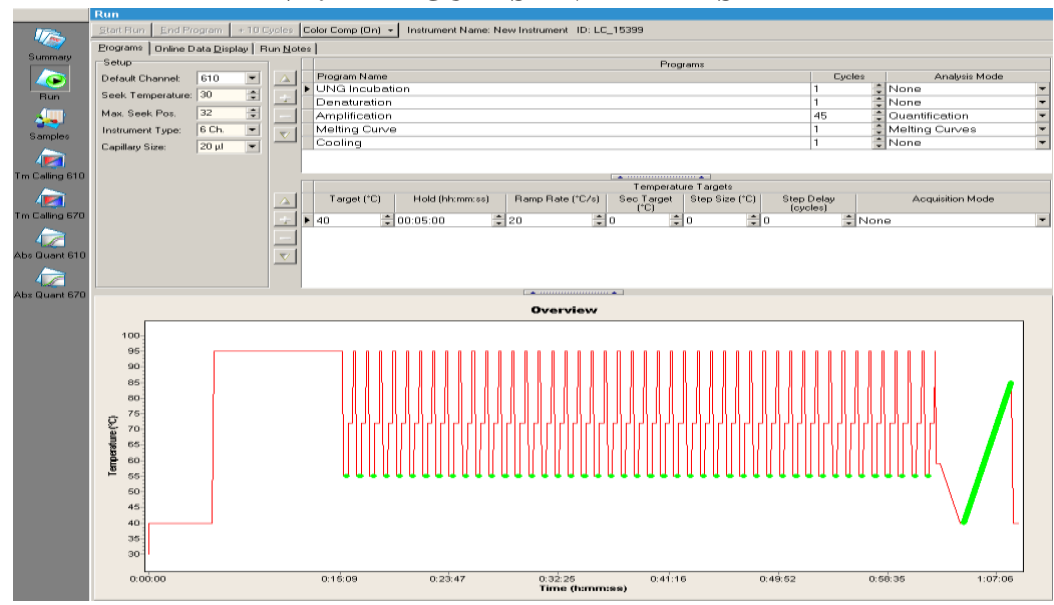

Figure 1. The cycling protocol consisted of one 10 minute cycle at $95^{\circ} \mathrm{C}$ followed by 45 cycles consisting of denaturation for 10 seconds at $95^{\circ} \mathrm{C}$, annealing for 10 seconds at $55^{\circ} \mathrm{C}$, and elongation for 15 seconds at $72^{\circ} \mathrm{C}$. The temperature ramp rate was set at $20^{\circ} \mathrm{C}$ per second. After the final cycle, the capillaries were cooled for $2 \mathrm{~min}$ at $40^{\circ} \mathrm{C}$.
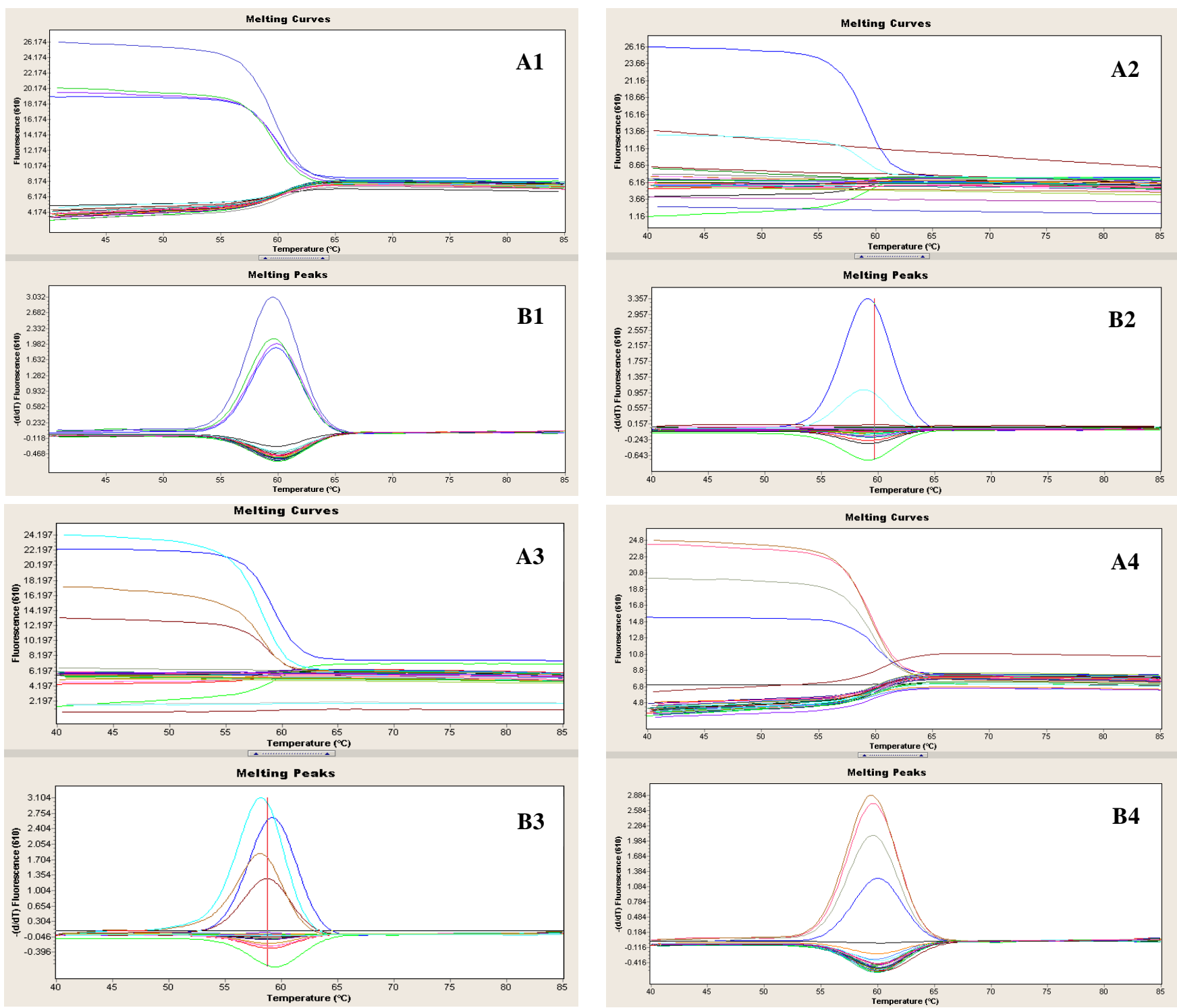

Figure 2. (A1-4) Fluorescence versus cycle number curves for the runs containing the 10 MRSA positive clinical samples. (B1-4) Melting point peaks for the samples in panel A showing differing melting temperatures $\left(\mathrm{T}_{\mathrm{M}}\right)$. In all plots, the positive and negative controls are represented by navy blue and lime green lines, respectively. Precise $\mathrm{T}_{\mathrm{M}}$ peak figures are shown in Table 1. 
Detection and simultaneous confirmation of Methicillin-Resistant Staphylococcus aureus by an

\begin{tabular}{|c|c|c|c|c|c|c|c|c|c|c|c|c|c|c|c|c|}
\hline $\begin{array}{c}\text { Isolat } \\
\text { e }\end{array}$ & $\mathbf{F}$ & $\begin{array}{c}\text { Ox } \\
\text { MIC* }\end{array}$ & $\mathbf{E}$ & DA & CIP & FD & MP & PEN & $\begin{array}{c}\text { SX } \\
\text { T }\end{array}$ & RD & $\begin{array}{c}\text { TE } \\
\mathbf{T}\end{array}$ & $\mathrm{CN}$ & $\begin{array}{l}\mathbf{T m} \\
\left({ }^{\circ} \mathbf{C}\right)\end{array}$ & $\begin{array}{l}s p a \\
\text { type }\end{array}$ & Strain & $\begin{array}{c}\text { SCCme } \\
c \text { type }\end{array}$ \\
\hline 1 & $\mathrm{R}$ & 32 & $S$ & $S$ & S & S & $S$ & $\mathrm{R}$ & $S$ & S & S & $\mathrm{S}$ & 58.1 & t002 & AK3 & IV \\
\hline 2 & $\mathrm{R}$ & 32 & $\mathrm{R}$ & $\mathrm{R}$ & S & S & S & $\mathrm{R}$ & $\mathrm{S}$ & S & S & $\mathrm{S}$ & 58.1 & $\mathrm{t} 002$ & AK3 & IV \\
\hline 3 & $\mathrm{R}$ & 4 & S & S & $S$ & $S$ & $S$ & $\mathrm{R}$ & $\mathrm{S}$ & S & S & $\mathrm{S}$ & 58.7 & t5677 & $\mathrm{N} / \mathrm{S}$ & - \\
\hline 4 & $\mathrm{R}$ & 4 & S & S & $S$ & S & S & $\mathrm{R}$ & $\mathrm{S}$ & S & S & S & 59.6 & t019 & WSPP & IV \\
\hline 5 & $\mathrm{R}$ & 4 & $S$ & $S$ & $S$ & $S$ & $S$ & $\mathrm{R}$ & $\mathrm{S}$ & S & $S$ & $\mathrm{~S}$ & 58.9 & t5677 & $\mathrm{N} / \mathrm{S}$ & - \\
\hline 6 & $\mathrm{R}$ & 64 & $S$ & $S$ & $S$ & $S$ & $S$ & $\mathrm{R}$ & $\mathrm{S}$ & S & $S$ & $S$ & 59.6 & t019 & WSPP & IV \\
\hline 7 & $\mathrm{R}$ & 64 & S & S & S & S & S & $\mathrm{R}$ & $\mathrm{S}$ & S & S & $\mathrm{S}$ & 59.6 & t019 & WSPP & IV \\
\hline 8 & $\mathrm{R}$ & 64 & S & S & S & S & S & $\mathrm{R}$ & $\mathrm{S}$ & S & S & $\mathrm{S}$ & 58.7 & t5677 & $\begin{array}{c}\mathrm{N} / \mathrm{S} \\
\mathrm{WR} / \mathrm{AK}\end{array}$ & - \\
\hline 9 & $\mathrm{R}$ & 32 & S & S & S & $\mathrm{R}$ & $\mathrm{R}$ & $\mathrm{R}$ & $\mathrm{S}$ & S & S & $\mathrm{S}$ & 57.7 & $\mathrm{t} 127$ & $\begin{array}{c}1 \\
\text { WR/AK }\end{array}$ & IV \\
\hline 10 & $\mathrm{R}$ & 32 & $\mathrm{~S}$ & $\mathrm{~S}$ & $\mathrm{~S}$ & $\mathrm{R}$ & $\mathrm{R}$ & $\mathrm{R}$ & $\mathrm{S}$ & S & $\mathrm{S}$ & $S$ & 57.7 & $\mathrm{t} 127$ & 1 & IV \\
\hline
\end{tabular}

Table 1. Antimicrobial susceptibility patterns (determined previously by Molan et al. [17]), melting temperatures $\left(\mathrm{Tm},{ }^{\circ} \mathrm{C}\right)$, spa types, MRSA strains, and SCCmec types of the 10 MRSA isolates detected by the

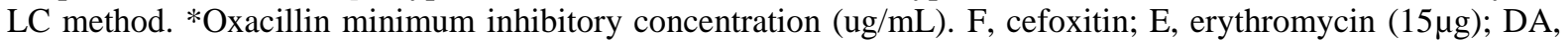

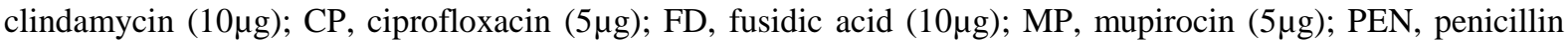
$(10 \mu \mathrm{g})$; SXT, co-trimoxazole $(25 \mu \mathrm{g})$; RD, rifampicin $(5 \mu \mathrm{g})$; TET, tetracycline $(30 \mu \mathrm{g})$; $\mathrm{CN}$, gentamicin $(10 \mu \mathrm{g})$. $\mathrm{N} / \mathrm{S}$, no identifiable strain.

\begin{tabular}{lr}
\hline Time for assay/test & Mean time \pm SD \\
\hline Roche LightCycler MRSA Advanced Test (PCR) & \\
Lysate preparation & $27 \pm 3.7 \mathrm{~min}$ \\
Master mix preparation & $2.6 \pm 0.3 \mathrm{~min}$ \\
PCR preparation & $20 \pm 3.0 \mathrm{~min}$ \\
Amplification and detection & $79.0 \pm 2.4 \mathrm{~min}$ \\
Total hands on time per run & $49.4 \pm 5.2 \mathrm{~min}$ \\
Total time per run (time to result) & $2.14 \pm 0.1 \mathrm{hours}$ \\
Average hands on time per specimen & $\mathbf{1 . 6 5} \pm \mathbf{0 . 1 7} \mathbf{~ m i n}$ \\
\hline
\end{tabular}

Table 2. Mean processing times and time to result of the PCR assay for a batch of 30 samples. Adapted from Molan et al. [17].

\section{REFERENCES}

[1] M Kuehnert, D Kruszon-Moran, H Hill, G McQuillan, S McAllister, G Fosheim, et al. Prevalence of Staphylococcus aureus Nasal Colonization in the United States, 2001-2002. Journal of Infectious Diseases, 193(2), 2006, 172-179.

[2] F DeLeo, H Chambers. Reemergence of antibiotic-resistant Staphylococcus aureus in the genomics era. Journal of Clinical Investigation, 119, 2009, 2464-2471.

[3] G Ayliffe. The Progressive Intercontinental Spread of Methicillin-Resistant Staphylococcus aureus. Clinical Infectious Diseases, 24, 1997, S74-79.

[4] J Engemann, Y Carmeli, S Cosgrove, V Fowler, M Bronstein, S Trivette, et al. Adverse Clinical and Economic Outcomes Attributable to Methicillin Resistance among Patients with Staphylococcus aureus Surgical Site Infection. Clinical Infectious Diseases, 36(5), 2003, 592-598.

[5] R Klevens, M Morrison, J Nadle, S Petit, K Gershman, S Ray, et al. Invasive Methicillin-Resistant Staphylococcus aureus Infections in the United States. Journal of the American Medical Association, 298(15), 2007, 1763-1771.

[6] A Larsen, M Stegger, S Bo"cher, M Sørum, D Monnet, R Skov. Emergence and Characterization of Community-Associated Methicillin-Resistant Staphyloccocus aureus Infections in Denmark, 1999 to 2006. Journal of Clinical Microbiology, 47(1), 2009, 73-78.

[7] S Cosgrove, G Sakoulas, E Perencevich, M Schwaber, A Karchmer, Y Carmeli. Comparison of mortality associated with methicillin-resistant and methicillin-susceptible Staphylococcus aureus bacteremia: a meta-analysis. Clinical Infectious Diseases, 36(1), 2003, 53-9.

[8] H Heffernan, S Bakker. Annual survey of methicillin resistant Staphylococcus aureus (MRSA), 2012. ESR Annual Report. Retrieved January 21, 2014, from: http://www.surv.esr.cri.nz

[9] F DeLeo, M Otto, B Kreiswirth, H Chambers. Community-associated meticillin-resistant Staphylococcus aureus. Lancet, 375 , 2010, 1557-68

[10] K Santos, L Tixeira, G Leal, L Fonseca, P Gontijo. DNA typing of methicillin-resistant Staphylococcus aureus: isolates and factors associated with nosocomial acquisition in two Brazilian university hospitals. Journal of Medical Microbiology, 48, 1999, 17-23.

[11] T Bannerman, G Hancock, F Tenover, J Miller. Pulse-field gel electrophoresis as a replacement for bacteriophage typing of Staphylococcus aureus. Journal of Clinical Microbiology, 33, 1995, 551-555.

[12] M Baddour, M Abuelkheir, A Fatani, M Bohol, M As-Ahdal. Molecular epidemiology of methicillin-resistant Staphylococcus aureus (MRSA) isolates from major hospitals in Riyadh, Saudi Arabia. Canadian Journal of Microbiology, 53, 2007, 931-936. 
[13] M Struelens, P Hawkey, G French, W Witte, E Tacconelli. Laboratory tools and strategies for methicillin-resistant Staphylococcus aureus screening, surveillance and typing: state of the art and unmet needs. Clinical Microbiology and Infection, 15, $2009,112-129$.

[14] L Peterson, O Liesenfeld, C Woods, S Allen, D Pombo, P Patel, et al. Multicenter Evaluation of the LightCycler MethicillinResistant Staphylococcus aureus (MRSA) Advanced Test as a Rapid Method for Detection of MRSA in Nasal Surveillance Swabs. Journal of Clinical Microbiology, 48(5), 2010, 1661-1666.

[15] E Sturenburg. Rapid detection of methiclllin resistant Staphylococcus aureus directly from clinical samples: methods, effectiveness and cost considerations. German Medical Science, 7, 2009, Doc06.

[16] B Strommenger, C Braulke, D Heuck, C Schmidt, B Pasemann, U Nübel, et al. spa typing of Staphylococcus aureus as a frontline tool in epidemiological typing. Journal of Clinical Microbiology, 46, 2008, 574-581.

[17] A Molan, M Nulsen, G Thomas. Methicillin-resistant Staphylococcus aureus (MRSA): isolation from nasal and throat swabs transported in liquid or semisolid media; identification by PCR compared with culture. New Zealand Journal of Medical Laboratory Science, 67, 2013, 8-16.

[18] J Smith, G Cook. A Decade of Community MRSA in New Zealand. Epidemiology and Infection, 133(5), 2005, 899-904

[19] A Richardson, U Desai, E Mowat, E Higgins, H Heffernan. Annual survey of methicillin resistant Staphylococcus aureus (MRSA), 2010. ESR Annual Report. Retrieved January 21, 2014, from: http://www.surv.esr.cri.nz

[20] G Coombs, G Nimmo, J Bell, F Huygens, F O’Brien, M Malkowski, J Pearson, A Stephens, P Giffard. Genetic diversity among community methicillin-resistant Staphylococcus aureus strains causing outpatient infections in Australia. Journal of Clinical Microbiology, 42, 2004, 4735-4743.

[21] J Chan, S Seet, F Chung. Methicillin-Resistant Staphylococcus aureus Strains from Singapore Show Shifted Melting Peaks in the LightCycler MRSA Advanced Test. Poster Abstract Session: Diagnostic Microbiology, presented at the ID week, Philadelphia, Thursday, October 18, 2012.

[22] J Boyce, N Havill. Comparison of BD GeneOhm Methicillin-Resistant Staphylococcus aureus (MRSA) PCR versus CHROMagar MRSA Assay for screening Patients for the Presence of MRSA strains. Journal of Clinical Microbiology, 46, 2008, $350-351$.

[23] C Valle, M Pasca, D De Vitis, F Marzani, V Emmi, P Marone. Control of MRSA infection and colonisation in an intensive care unit by GeneOhm MRSA assay and culture methods. BMC Infectious Diseases, 9, 2009137.

[24] M Holfelder, U Eigner, A Turnwald, W Witte, M Weizenegger, A Fahr, A. Direct detection of methicillin-resistant Staphylococcus aureus in clinical specimens by a nucleic acid-based hybridisation assay. Clinical Microbiology and Infection, 12, 2006, 163-7.

[25] R Cunningham, P Jenks, J Northwood, M Wallis, S Ferguson, S Hunt. Effect on MRSA transmission of rapid PCR testing of patients admitted to critical care. Journal of Hospital Infections, 65, 2007 24-28. 